\title{
Comparison of Voiding Questionnaires Between Female Interstitial Cystitis and Female Idiopathic Overactive Bladder
}

\author{
Sung-Han Kim, Seung-June Oh \\ Department of Urology, Seoul National University Hospital, Seoul, Korea
}

Purpose: This study aimed to find out any different characteristics in various different voiding symptom questionnaires in the outpatient clinics between interstitial cystitis and overactive bladder.

Materials and Methods: Between October 2005 and December 2007, retrospectively were analyzed the consecutive 41 IC female patients' and $43 \mathrm{OAB}$ female patients' questionnaires, who had completed three questionnaires at the outpatient department (Incontinence Quality of Life; I-QoL, King's Health Questionnaire; KHQ, International Prostate Symptom Score; IPSS). Additionally, the 41 IC patients also completed O'Leary Sant Questionnaire (OLS, or ICSI/ICPI).

Results: No statistical differences existed in age and symptom duration between two groups ( $\mathrm{p}>0.05)$. In I-QoL, only the social embarrassment score was statistically different between two groups $(\mathrm{p}<0.05)$, but the total score, avoidance and limiting behavior, and psychosocial impact scores were not $(\mathrm{p}>0.05)$. In KHQ, the general health perceptions, impact on life, social limitations, personal relationships, emotions, and sleep/energy scores were statistically different $(\mathrm{p}<0.033)$, but the role limitations, physical limitations, and incontinence severity measures scores were not $(p>0.059)$. In IPSS, the straining symptom, and quality of life scores were different between two groups $(\mathrm{p}<0.05)$. The IC patients also completed OLS questionnaire with mean interstital cystitis symptom index score of $14.10 \pm 3.92$, and interstitial cystitis problem index score of $11.79 \pm 3.75$.

Conclusions: This study showed that some differences among symptom questions in questionnaires did exist between the two groups and that the IC group had much more impaired quality of life than the OABs.

Key Words: Interstitial cystitis; Overactive bladder; Questionnaire

\section{Introduction}

Clinically, under the broad definition of interstitial cystitis (IC), interstitial cystitis/painful bladder syndrome (IC/PBS) is another name for severe, painful voiding symptoms without any bacterial infections or specific underlying causes. IC/PBS is ultimately diagnosed on the basis of the voiding symptoms after ruling out other pathologically caused diseases [1]. Overactive bladder $(\mathrm{OAB})$ is diagnosed on the basis of voiding symptoms such as frequency, urgency, nocturia, and sometimes urge incontinence [2]. However, these two diseases have such similar voiding and associated symptoms that clinical difficulties in diagnosis are a common problem for clinicians [3,4].

Therefore, with the use of various questionnaires on voiding symptoms that are fre-

\footnotetext{
Corresponding Author: Seung-June Oh

Department of Urology, Seoul National University Hospital, 28, Yeongeon-dong, Jongno-gu, Seoul 110-744, Seoul, Korea

Tel: +82-2-760-2406 / Fax: +82-2-742-4665 / E-mail: sjo@snu.ac.kr
}

Submitted: July 09, 2010 / Accepted (with revisions): August 19, 2010 
quently used clinically, such as the Incontinence Quality of Life (IQoL), King's Health Questionnaire (KHQ), International Prostate Symptom Score (IPSS), and O'Leary Sant Questionnaire (OLS, or ICSI/ICPI), this study aimed to compare characteristics of the quality of life of patients with IC or $\mathrm{OAB}$ in order to better understand the diseases and the patients' lives.

\section{Materials and Methods}

Between October 2005 and December 2007, according to the International Continence Society (ICS) 2002 [5], the outpatient charts of 45 consecutive IC female patients and 170 idiopathic $\mathrm{OAB}$ female patients were analyzed retrospectively. Ultimately, 45 IC patients and 43 idiopathic OAB patients who had completed three questionnaires at the outpatient department (IQoL, KHQ, and IPSS) were enrolled in this study. Additionally, the 41 IC patients completed the OLS (or ICSI/ICPI).

All IC and idiopathic OAB patients completed the voiding questionnaires before the completion of their first examination at the outpatient clinic. They underwent the previously ordered routine examination, including history taking, physical examination, urine analysis, uroflowmetry, post-voided residual volume, and gynecological screening by gynecologists. On the second visit, they were asked to submit their 3-day voiding diaries and then to undergo some more invasive studies, such as the urodynamic study, diagnostic cystoscopy, and potassium sensitivity test, which were performed for all IC patients. All the patients in this study were over 20 years old and had no history of previous radiation therapy, urological operations, stress incontinence, acute urinary infection, pregnancy, cancer, dementia or other neurologic disease, uncontrolled diabetic neuropathy, spinal cord injury or cerebrovascular diseases, bleeding tendency disease, psychological disease, degenerative disease, or metabolic disease. The 41 IC patients met the criteria of the National Institute of Diabetes, Digestive and Kidney Disease (NIDDK) [6] after thoroughly finishing the examinations.

This retrospective study was approved by the IRB, and the data were analyzed by use of SPSS v12.0. The Mann-Whitney test and chi-square test were used to statistically compare the contents and scores of the IQoL, KHQ, IPSS, and OLS questionnaires between the IC and OAB groups. Statistical analysis was performed by the Medical Research Collaboration Center of the Seoul National University Hospital.

\section{Results}

No statistical differences existed in mean age or median symptom duration between the IC and OAB groups $(p>0.05)$. On the IQoL, only the social embarrassment score was statistically different between the two groups $(p=0.003)$, whereas the total scores, avoidance and limiting behavior scores, and psychosocial impact scores were not ( $p>0.05$, Table 1, demographics of IC and OAB patients). On the $\mathrm{KHQ}$, among the 9 questions concerning quality of life, the total scores of the IC group were generally higher than those of the $\mathrm{OAB}$ group except for the general health perception scores and incontinence severity measure scores, for which the scores of the OAB group were higher (Table 1). The scores for general health perceptions, impact on life, social limitations, personal relationships, emotions, and sleep/energy were statistically different $(p<0.033)$, but the scores for role limitations, physical limitations, and incontinence severity measures were not $(p>0.059)$. There were also statistically significant differences in scores for the general health perceptions $(p=0.033)$, impact on life $(p=0.014)$, social limitations $(p=0.030)$, personal relationships $(p=0.032)$, emotions $(p=0.004)$, and sleep/energy $(p=0.011)$ questions between the two groups. Among the 10 additional KHQ symptom questions, there were some statistically significant differences in the scores of the urge incontinence $(p=0.004)$, stress incontinence $(p=0.003)$, bladder pain $(p=0.003)$, and voiding difficulty $(p=0.009)$ questions between the two groups. For the IPSS, the total score was higher in the IC group than in the OAB group (Table 2, IPSS questionnaire: IC vs OAB). Except for the intermittency and urgency questions, the IC group had higher scores than the $\mathrm{OAB}$ group for the rest of the voiding symptoms, but only the scores for the straining 
Table 1. Demographics of IC patients and OAB patients

\begin{tabular}{|c|c|c|c|}
\hline & $\begin{array}{l}\text { Interstitial Cystitis } \\
(\mathrm{N}=41) \\
\text { Mean }( \pm \mathrm{SD})\end{array}$ & $\begin{array}{l}\text { Overactive Bladder } \\
\qquad(\mathrm{N}=43) \\
\text { Mean }( \pm \mathrm{SD})\end{array}$ & $P$ value \\
\hline Age (mean $\pm \mathrm{SD}$, months) & $58.2( \pm 13.2)$ & $61.9( \pm 13.5)$ & $0.217^{+}$ \\
\hline Median duration of symptom (interval range, months) & $38(12-80)$ & $16(12-21)$ & $0.602^{+}$ \\
\hline \multicolumn{4}{|l|}{ Incontinence Quality of Life questionnaire ${ }^{++}$} \\
\hline Total score & $54.8( \pm 24.0)$ & $52.6( \pm 20.3)$ & 0.692 \\
\hline Avoidance and limiting behavior score & $54.6( \pm 23.3)$ & $51.5( \pm 17.4)$ & 0.544 \\
\hline Psychosocial impacts score & $60.5( \pm 30.1)$ & $47.5( \pm 24.3)$ & 0.061 \\
\hline Social embarrassment score & $45.2( \pm 23.4)$ & $63.8( \pm 23.9)$ & 0.003 \\
\hline \multicolumn{4}{|l|}{ King's Health questionnaire ${ }^{+++}$} \\
\hline General health perceptions score & $16.1( \pm 13.8)$ & $24.2( \pm 15.9)$ & 0.033 \\
\hline Impact on life score & $60.2( \pm 13.4)$ & $50.5( \pm 16.9)$ & 0.014 \\
\hline Role limitations score & $54.3( \pm 16.7)$ & $46.5( \pm 17.6)$ & 0.072 \\
\hline Physical limitations score & $53.2( \pm 20.4)$ & $43.4( \pm 20.4)$ & 0.059 \\
\hline Social limitations score & $46.4( \pm 26.5)$ & $32.6( \pm 22.7)$ & 0.030 \\
\hline Personal relationships s score & $46.3( \pm 25.5)$ & $30.6( \pm 25.4)$ & 0.032 \\
\hline Emotions score & $51.6( \pm 18.7)$ & $37.4( \pm 19.2)$ & 0.004 \\
\hline Sleep/energy score & $48.9( \pm 21.9)$ & $35.4( \pm 19.0)$ & 0.011 \\
\hline Incontinence severity measures score & $27.1( \pm 20.4)$ & $34.6( \pm 15.7)$ & 0.110 \\
\hline \multicolumn{4}{|l|}{ Urinary symptoms score } \\
\hline Frequency & $2.63( \pm 0.76)$ & $2.31( \pm 0.82)$ & 0.117 \\
\hline Nocturia & $2.50( \pm 0.97)$ & $2.06( \pm 0.98)$ & 0.083 \\
\hline Urgency & $2.00( \pm 1.20)$ & $2.16( \pm 1.08)$ & 0.592 \\
\hline Urge incontinence & $0.93( \pm 1.08)$ & $1.81( \pm 1.20)$ & 0.004 \\
\hline Stress incontinence & $0.80( \pm 1.00)$ & $1.72( \pm 1.28)$ & 0.003 \\
\hline Enuresis & $0.43( \pm 0.90)$ & $0.66( \pm 0.90)$ & 0.334 \\
\hline Sexual incontinence & $0.40( \pm 0.81)$ & $0.63( \pm 0.98)$ & 0.330 \\
\hline Recurrent cystitis & $1.57( \pm 1.33)$ & $1.25( \pm 1.30)$ & 0.346 \\
\hline Bladder pain & $2.10( \pm 1.27)$ & $1.13( \pm 1.24)$ & 0.003 \\
\hline Voiding difficulty & $1.80( \pm 1.27)$ & $0.97( \pm 1.14)$ & 0.009 \\
\hline
\end{tabular}

${ }^{+}$: Mann-Whitney test

${ }^{++}$: Calculated according to Patrick DL et al [8].

${ }^{+++}$: Calculated according to Okamura $\mathrm{K}$ et al [12].

symptom and quality of life questions were significantly different $(\mathrm{p}<0.05)$. The IC patients also completed the OLS questionnaire with a mean interstitial cystitis symptom index score of $14.10 \pm 3.92$ and a mean interstitial cystitis problem index score of $11.79 \pm 3.75$ (Table 3 , OLS ques- tionnaire: IC). For the ICSI, the highest scores (in consecutive order) were for bladder pain, frequency, nocturia, and urgency for the ICPI, the highest scores were for bladder pain, urgency, frequency, and nocturia. 
Table 2. International prostate symptom score questionnaire [16]: interstitial cystitis versus overactive bladder

\begin{tabular}{lccc}
\hline Variable & $\begin{array}{c}\text { Interstitial cystitis }(n=41) \\
\text { Mean }( \pm S D)\end{array}$ & $\begin{array}{c}\text { Overactive bladder }(n=43) \\
\text { Mean }( \pm S D)\end{array}$ \\
\hline 1. Incomplete emptying & $2.71( \pm 1.89)$ & $2.68( \pm 1.99)$ & 0.943 \\
2. Frequency & $3.93( \pm 1.63)$ & $3.22( \pm 1.73)$ & 0.066 \\
3. Intermittency & $1.93( \pm 1.85)$ & $2.19( \pm 2.03)$ & 0.552 \\
4. Urgency & $3.35( \pm 1.90)$ & $3.49( \pm 1.84)$ & 0.750 \\
5. Weak stream & $2.73( \pm 1.99)$ & $2.45( \pm 2.10)$ & 0.559 \\
6. Straining & $2.24( \pm 1.98)$ & $1.35( \pm 1.98)$ & 0.050 \\
7. Nocturia & $3.05( \pm 1.66)$ & $2.43( \pm 1.39)$ & 0.081 \\
Total symptom score & $2.37( \pm 0.70)$ & $2.16( \pm 0.76)$ & 0.223 \\
8. Quality of life due to urinary symptoms & $5.17( \pm 1.14)$ & $4.38( \pm 1.28)$ & 0.005 \\
\hline
\end{tabular}

Table 3. O'Leary Sant questionnaire [13]: Interstitial cystitis (total 34 patients

\begin{tabular}{lcc}
\hline & $\begin{array}{c}\text { Interstitial cystitis symptom indices } \\
\text { Mean }( \pm \text { SD) }\end{array}$ & $\begin{array}{c}\text { Interstitial cystitis problem indices } \\
\text { Mean }( \pm \text { SD) }\end{array}$ \\
\hline Total score & $14.10( \pm 3.92)$ & $11.79( \pm 3.75)$ \\
Urgency & $2.97( \pm 1.82)$ & $3.03( \pm 1.15)$ \\
Frequency & $3.83( \pm 1.65)$ & $2.90( \pm 1.29)$ \\
Nocturia & $3.48( \pm 1.33)$ & $2.52( \pm 1.66)$ \\
Bladder pain & $3.72( \pm 1.58)$ & $3.17( \pm 1.34)$ \\
\hline
\end{tabular}

\section{Discussion}

OAB has many similar voiding symptoms to IC, such as frequency, nocturia, and urgency with or without urge incontinence thus, it is not easy to differentiate $\mathrm{OAB}$ from IC because of the clinically overlapping features [3,4]. Like $\mathrm{OAB}$, IC is associated with bladder pain in particular, but it has a variety of symptoms related to the clinical stage, such that in early IC, the urgency-like symptoms might appear like OAB [3]. However, the IC patients in the present study satisfied the classifications of both the International Continence Society (ICS) 2002 [5], which were made for clinicians to easily adapt for diagnosis, and the NIDDK [6], which were designed for in- vestigational study. The patients had a mean IC history of at least 12 months and had suffered from severe bladder pain according to their total scores on the OLS questionnaires. Therefore, we can conclude that the IC and OAB patients were clearly differentiated.

The IQoL is a psychometric performance selfreported questionnaire made for female incontinent patients to evaluate their quality of life relating to incontinence [7]. It is an internationally used questionnaire in which each spectrum of questions can be calculated from 0 to 100 higher scores are interpreted to indicate a better quality of life [8]. In this study, the IC group had statistically insignificantly higher scores on the IQoL for the total score, avoidance and limiting behavior, and psychosocial impact score, except for the 
social embarrassment score. The social embarrassment score was statistically significantly lower in the IC group than in the OAB group. This suggests that, according to the previously reported interpretations of lower social embarrassment domain scores indicating a poorer quality of life, the IC patients had a more impaired quality of life than did the OAB patients as a result of their disease [9]. This helps to explain why IC patients become more hypersensitive to influences of the disease on their quality of life than do patients with OAB.

The KHQ is an internationally popular questionnaire for the evaluation of disease-specific, health-related quality of life and has 9 spectra that are objectively scored from 0 to $100[10,11]$. It has 10 additional symptom-related severity questions, and higher scores are interpreted to mean a poorer quality of life within the spectrum [12]. In this study, the scores for urge incontinence and stress incontinence in the $\mathrm{OAB}$ group and for bladder pain and voiding difficulty in the IC group were statistically significantly higher than in the other group. These results are consistent with the differentiating symptoms between these two diseases. Among the 9 questions about quality of life, except for the higher general health perception score and incontinence severity measures score in the OAB group, the IC patients had generally higher total scores than did the OAB patients. However, among the 9 spectra, the statistically significantly different spectra in the IC patients were the scores for general health perceptions, impact on life, social limitations, personal relationships, emotions, and sleep/energy, which were influenced by the disease such that the quality of life of the IC patients was much poorer than that of the $\mathrm{OAB}$ patients. According to the KHQ results, the IC patients were not energetic and were unable to sleep well, and their emotions were poorly affected by their voiding problems so that they had limited social behaviors and personal relationships, and all of these impacts resulted in a poorer quality of life. The reason the general health perception scores of the $\mathrm{OAB}$ patients were higher was because the KHQ voiding questionnaire was perhaps more related to the quality of life of incontinent people.
The IPSS is composed of 8 questions concerning benign prostatic hyperplasia and various other lower urinary tract symptoms, including quality of life [13]. Comparing the first self-reported IPSS questionnaires in both the IC and OAB groups, except for the question on intermittency, all other questions and total scores were higher in the IC group than in the OAB group, but only the questions on straining and quality of life were statistically significant. One explanation for this result in the present study with the IPSS questionnaire is that the IC and OAB groups had similar and overlapping symptoms except for pain and urge incontinence, which is the same conclusion as in previous studies [3,4]. IC patients with bladder pain can be suggested to have a poorer quality of life as well as straining voiding symptoms compared with OAB patients.

The OLS is auseful questionnaire made for IC patients to objectively evaluate the severity of symptoms, diagnosis, and effects of treating responsiveness and is used throughout the world [14]. Among the 41 IC patients, only 34 patients properly completed the OLS questionnaire. According to previously performed studies with the OLS, total scores on the ICSI and ICPI over 6 points were used as the diagnostic baseline criteria in IC screening, and further evaluation was suggested to be performed for making a diagnosis [15]. In other previous studies, ICSI or ICPI total scores over 12 points were suggested to indicate severe forms of IC [16]. These remarks also explain our results of high scores of 14.10 and 11.79 for the ICPI and ICPI, which indicates that our IC participants had severe forms of IC. The higher scores for the consecutive symptom sequence of bladder pain and frequency on the ICSI and the higher scores for bladder pain and urgency on the ICPI indicate that the IC patients in our study complained chiefly of bladder pain and frequency. However, our study shows for the first time that IC patients suggest that bladder pain and urgency be the first treated symptoms above all according to their influential effect on quality of life. We conclude that this means that bladder pain and its related symptoms were treated more actively than other symptoms.

This study had a short-term period with retro- 
spective observation of a small number of patients and might have a selection bias. However, these patients received consistent examinations according to previously set protocols in a specific period and only the analysis was performed retrospectively. It is therefore possible that selection bias did not exist. Further long-term study might be necessary with a large number of patients. Secondly, this study used the KHQ and IQoL questionnaires, which have not been validated in IC. However, the KHQ and IQoL have been used and validated throughout the world in various disease patients, and a recent study with the IQoL was performed in IC patients to evaluate quality of life [17]. It is meaningful to compare the quality of life between $\mathrm{OAB}$ and IC patients, who have similar clinical symptoms, so that the patients can evaluate the influence of the disease on their lives. The disease spectrum of IC is wide and very different at each stage of the disease. The present study used the questionnaires at a specific period of time. Therefore, we could not evaluate the effect of clinical stage on quality of life. Additional validation of the KHQ and IQoL is necessary, and some useful and effective questionnaires about the quality of life in IC are needed.

\section{Conclusions}

After thoroughly comparing the questionnaires between the IC and OAB groups, we showed that some differences among symptom questions did exist and that the IC group had much poorer scores on questions about their quality of life than did the OAB group.

Therefore, the results of this study have shown that voiding questionnaires may be helpful tools for clinicians to better understand the comparative quality of life in both groups of patients.

\section{References}

1) Hanno PM. Painful bladder syndrome/interstitial cystitis and related disorder. In: Wein AJ, Kavoussi LR, Novick AC, Partin AW, Peters CA, editors. Campbell-Walsh urology, 9th ed. Philadelphia: Saunders; 2007;331-3

2) Abrams P, Drake M. Overactive bladder. In: Wein
AJ, Kavoussi LR, Novick AC, Partin AW, Peters CA, editors. Campbell-Walsh urology, 9th ed. Philadelphia: Saunders; 2007;2079-84

3) Abrams P, Hanno P, Wein A. Overactive bladder and painful bladder syndrome: There need not be confusion. Neurourol Urodyn 2005;24:149-50

4) Clemens JQ, Markossian TW, Meenan RT, O'Keeffe Rosetti MC, Calhoun EA. Overlap of voiding symptoms, storage symptoms and pain in men and women. J Urol 2007;178:1354-8

5) Abrams P, Cardozo L, Fall M, Griffiths D, Rosier P, Ulmsten $\mathrm{U}$, et al. The standardisation of terminology in lower urinary tract function: report from the standardisation sub-committee of the International Continence Society. Urology 2003;61:37-49

6) Gillenwater JY, Wein AJ. Summary of the national institute of arthritis, diabetes, digestive and kidney diseases workshop on interstitial cystitis. National Institutes of Health, Bethesda, Maryland, August 28-29, 1987. J Urol 1988;140:203-6

7) Oh SJ, Park HK, Lim SH, Hong SK, Martin ML, Ting BL, et al. Validation of Korean version of self-written Incontinence Quality of Life (IQOL). J Korean Continence Soc 2002;6:10-23

8) Patrick DL, Martin M, Bushnell DM. The I-QOL, user's manual and scoring diskette. Seattle, WA, U.S.A.: Health Research Associates, Inc.

9) McKenna SP, Whalley D, Renck-Hooper U, Carlin S, Doward LC. The development of a quality of life instrument for use with post-menopausal women with urogenital atrophy in the UK and Sweden. Qual Life Res 1999;8:393-8

10) Kelleher CJ, Cardozo LD, Khullar V, Salvatore S. A new questionnaire to assess the quality of life of urinary incontinent women. Br J Obstet Gynaecol 1997;104:1374-9

11) Oh SJ, Choo MS, Kim HS, Kim JC, Lee JG, Yoon $\mathrm{JM}$, et al. Psychometric properties of Korean version of the King's Health Questionnaire in women with stress urinary incontinence. J Korean Continence Soc 2005;9:115-23

12) Okamura $K$, Usami $T$, Nagahama $K$, Maruyama $S$, Mizuta E. "Quality of life" assessment of urination in elderly Japanese men and women with some medical problems using International Prostate Symptom Score and King's Health Questionnaire. Eur Urol 2002;41:411-9

13) O'Leary MP, Sant GR, Fowler FJ Jr, Whitmore KE, Spolarich-Kroll J. The interstitial cystitis symptom index and problem index. Urology 1997;49:58-63

14) Lubeck DP, Whitmore K, Sant GR, Alvarez-Horine S, Lai C. Psychometric validation of the O'learySant interstitial cystitis symptom index in a clinical 
trial of pentosan polysulfate sodium. Urology 2001;57:62-6

15) Leppilahti M, Tammela TL, Huhtala $H$, Auvinen A. Prevalence of symptoms related to interstitial cystitis in women: a population based study in Finland. J Urol 2002;168:139-43

16) Cockett A, Aso Y, Denis L. Prostate symptom score and quality of life assessment. In: Cockett ATK, Khoury S, Aso Y, Chatelain C, Denis L, Griffiths K, et al, editors. Proceedings of the Second International Consultation on Benign Prostatic Hyperplasia (BPH); 27-30 June 1993; Paris; Channel Island, Jersey: Scientific Communication International; 1994;553-555

17) El Khoudary SR, Talbott EO, Bromberger JT, Chang CC, Songer TJ, Davis EL. Severity of interstitial cystitis symptoms and quality of life in female patients. J Womens Health 2009;18:1361-8 